\title{
Original
}

\section{The Early Autophagic Pathway Contributes to Osteogenic Differentiation of Human Periodontal Ligament Stem Cells}

\author{
Madoka Yasunaga $^{1,2)}$, Hiroshi Kajiya ${ }^{2,3)}$, Takuya Toshimitsu ${ }^{2,4)}$, Hiroki Nakashima ${ }^{1,2)}$, Sachio Tamaoki ${ }^{1)}$, Hiroyuki Ishikawa ${ }^{5)}$, \\ Hidefumi Maeda" and Jun Ohno ${ }^{2)}$ \\ ${ }^{1)}$ Section of Orthodontics, Department of Oral Growth and Development, Fukuoka Dental College, Fukuoka, Japan \\ ${ }^{2)}$ Research Center for Regenerative Medicine, Fukuoka Dental College, Fukuoka, Japan \\ ${ }^{3)}$ Section of Cellular Physiology, Department of Physiological Science and Molecular Biology, Fukuoka Dental College, Fukuoka, Japan \\ ${ }^{4)}$ Dentistry for the Disabled, Department of Oral Growth and Development, Fukuoka Dental College, Fukuoka, Japan \\ ${ }^{5)}$ Former Section of Orthodontics, Department of Oral Growth and Development, Fukuoka Dental College, Fukuoka, Japan \\ ${ }^{6}$ Division of Oral Rehabilitation, Department of Endodontology and Operative Dentistry, Faculty of Dental Science, Kyushu University, Fukuoka, \\ Japan \\ (Accepted for publication, December 19, 2018)
}

\begin{abstract}
This study aimed to investigate the role of autophagic stages through the interplay of AMP-activated protein kinase (AMPK)/mammalian target of rapamycin (mTOR)/Beclin-1 signaling pathways in osteogenic differentiation of human periodontal ligament stem cells (HPLSCs) using a combination of pharmacological inhibition and genetic knockdown approaches. The activation or inactivation of these factors and autophagy was evaluated by western blot and immunocytochemical assays, while osteogenic differentiation was analyzed by alkaline phosphatase staining. Enhancement of osteogenic differentiation of HPLSCs was correlated with the increased expression of the autophagy markers Beclin-1 and microtubule-associated protein 1 light chain 3-II (LC3-II), and phosphorylation of AMPK, consistent with reduced expression of phosphorylated p70S6 kinase, mTOR substrate. The RNA interference-mediated silencing of AMPK and Beclin-1 (marker of early autophagy) and the pharmacological inhibitors of AMPK (compound C) and early autophagy (3-methyladenine) suppressed acceleration of osteogenic differentiation and autophagy. mTOR inhibition increased both osteogenic differentiation and autophagy. Inhibition of the late stage of autophagy by LC3 siRNA and chloroquine resulted in no changes in the osteogenic differentiation of HPLSCs. These findings suggest that the early stages of autophagy through the AMPK/ mTOR/Beclin-1 signaling pathway may be required for the enhancement of osteogenic differentiation of HPLSCs.
\end{abstract}

Key words: Osteogenesis, Human periodontal ligament stem cells, Autophagy, AMPK, mTOR, Beclin-1

\section{Introduction}

Macroautophagy (hereafter referred to as autophagy) is an intracellular degradation process by which cytosolic materials, including damaged organelles and toxic protein aggregates, are sequestered in specialized double membrane-bound autophagosomes ${ }^{1,2)}$. Autophagy plays a role in the differentiation of erythrocytes, lymphocytes, and adipocytes ${ }^{3)}$. Previous studies have revealed that the induction of autophagy contributes to osteogenic differentiation of mesenchymal stem cells (MSCs) ${ }^{4,5}$.

The process of autophagosome formation is divided into four main steps: initiation/nucleation, elongation, maturation, and degradation ${ }^{3,6}$. In the early stages of the autophagic process, phagophore formation at the initiation phase and nucleation occur with the formation of class III phosphatidylinositol 3-kinase (PI3KcIII) complexes composed of the proteins Beclin-1, Vsp15, Vsp34, Ambra1, UVRAG, and others. Among these proteins, Beclin-1 is fundamental for the formation of PI3KcIII complexes, thus Beclin-1 is commonly used as a marker of the early stage of autophagy ${ }^{7,8)}$. After nucleation, phagophore elongation occurs

Correspondence to: Dr. Jun Ohno, Research Center for Regenerative Medicine, Fukuoka Dental College, 2-15-1 Tamura, Sawara-ku, Fukuoka, Fukuoka 8140193, Japan; Tel: +81928010411 (Ext 684); Fax: +81928014909;

E-mail: johno@college.fdcnet.ac.jp and an autophagosome is formed in the late stages of autophagy. At late autophagy, LC3-II is found within the autophagosome membrane and, therefore, has been widely used as a specific marker of the late stages of autophagy ${ }^{6,9)}$.

Human adult MSCs have the potential for differentiation into various cell types, including osteoblasts, chondrocytes, and adipocytes ${ }^{10,11)}$. In dentistry, suitable tooth-derived stem cell have been identified and characterized to evaluate in tissue engineering studies. Among tooth-derived stem cells, human periodontal ligament stem cells (HPLSCs) express a variety of stromal cell markers with similar morphological, phenotypic, and proliferative characteristics of adult $\mathrm{MSCs}^{12)}$, but have a greater potential to differentiate into osteoblasts and adipocytes ${ }^{13)}$. Hence, HPLSCs are considered as the most promising source of stem cells for periodontal regenerative therapies ${ }^{12-16)}$. Therefore, it is important to understand the molecular mechanisms underlying the osteogenic differentiation of HPLSCs. While it appears that the induction of autophagy is involved in the osteogenic differentiation of MSCs originating from various tissues, presently, there is no such data for HPLSCs. Additionally, the role of autophagic pathways in the osteogenic differentiation of HPLSCs remains unclear.

The autophagic process is regulated by several factors. Of these, 
adenosine monophosphate-activated protein kinase (AMPK) is a positive regulator of autophagy and acts as a principal intracellular energy sensor that induces autophagy mainly through phosphorylation of its downstream target Raptor and consequent inhibition of the serine/threonine kinase mammalian target of rapamycin $(\mathrm{mTOR})^{5,17)}$. Conversely, mTOR is a central regulator of cell growth and a negative regulator of autophagy. mTOR is also known to induce osteogenic differentiation of various cells ${ }^{18,19)}$. In this study, we investigated the role of autophagic stages through the interplay of the AMPK/mTOR/Beclin-1 signaling pathways in osteogenic differentiation of HPLSCs using a combination of pharmacological inhibition and genetic knockdown approaches.

\section{Materials and Methods \\ Reagents and antibodies (Abs)}

Dulbecco's modified Eagle's medium (DMEM) was purchased from Fujifilm WakoPure Chemical Co., (Osaka, Japan). Fetal bovine serum (FBS) was purchased from HyClone Laboratories Inc. (South Logan, UT, USA). $1 \times$ antibiotic-antimycotic solution $(1 \times$ Anti-Anti) was obtained from Invitrogen Corporation (Carlsbad, CA, USA). Rapamycin, 3-methyladenine (3-MA), chloroquine (CQ), Hoechst 33324 nucleic acid stain, an alkaline phosphatase (ALP) kit, and monoclonal Ab against $\beta$-actin were purchased from Sigma-Aldrich Corporation (St. Louis, MO, USA). The Pierce ${ }^{\mathrm{TM}} \mathrm{BCA}$ Protein Assay Kit and Lipofectamine ${ }^{\mathrm{TM}}$ RNAi MAX were obtained from Thermo Fisher Scientific (Rockford, IL, USA). Dorsomorphin (compound C, CC), rabbit anti-Sp7/osterix, and rabbit anti-runt-related transcription factor 2 (Runx2) were obtained from Abcam Inc. (Cambridge, UK). Rabbit Abs against microtubule-associated protein 1 light chain 3 (LC3) and Beclin-1 were purchased from MBL (Tokyo, Japan). Precision Plus Protein Western C Standard, $4 \%-20 \%$ and $12 \%$ Mini-PROTEAN ${ }^{\circledR}$ TGX $^{\mathrm{TM}}$ Precast Gels, Trans-Blot Transfer Packs, and horseradish peroxidase-conjugated anti-mouse and -rabbit secondary Abs were obtained from Bio-Rad Laboratories (Hercules, CA, USA). Cell lysis buffer, SignalFire ${ }^{\mathrm{TM}}$ Plus ECL Reagent, $1 \times$ protease/phosphatase inhibitor cocktail, rabbit anti-AMP$\mathrm{K} \alpha$, rabbit anti-phospho AMPK $\alpha$ (Thr172; p-AMPK), rabbit anti-p70S6 kinase (p70S6K), rabbit anti-phospho p70S6K (Thr389; p-p70S6K), rabbit phospho-mTOR (Ser2448, p-mTOR), SignalSilence LC3B siRNA, SignalSilence AMPK $\alpha$ siRNA, and SignalSilence Beclin-1 siRNA were purchased from Cell Signaling Technology (Danvers, MA, USA). mTOR siRNA was obtained from Santa Cruz Biotechnology (Dallas, TX, USA).

\section{Cell culture}

HPLSCs that were stored at the Department of Endodontology and Operative Dentistry, Division of Oral Rehabilitation, Faculty of Dental Science, Kyushu University ${ }^{14)}$, were cultured in DMEM supplemented with $10 \%(\mathrm{v} / \mathrm{v})$ FBS and $1 \times$ Anti-Anti solution. Upon reaching confluence, the cells were cultured in osteogenic induction medium (OIM), which was composed of DMEM supplemented with $10 \mathrm{nM}$ dexamethasone, $200 \mu \mathrm{M}$ ascorbic acid, $10 \mathrm{mM} \beta$-glycerophosphate, and 10\% FBS. The OIM was replaced three times each week.

\section{Cell transfection}

HPLSCs were placed in the wells of 12-well plates for $24 \mathrm{~h}$ before transfection. Transfection of siRNA-mTOR, AMPK $\alpha$, Beclin-1, and LC3 was conducted using $10 \mu \mathrm{M}$ of siRNAs per well and Lipofectamine RNAiMAX Transfection Reagent in accordance with the manufacturer's instructions. At $48 \mathrm{~h}$ post-transfection, the cells were cultured in OIM for 7 days.

\section{ALP staining}

HPLSCs were stained with an ALP Kit containing Fast Red Violet solution and naphthol AS-BI phosphate solution, in accordance with the manufacturer's instructions. Briefly, HPLSCs cultured with OIM for 7 days were fixed with citrate-acetone-formaldehyde for 30 seconds and rinsed in distilled water (DW). Cells were incubated with alkaline-dye mixture for $15 \mathrm{~min}$ and washed with DW. The reaction product was evaluated by microscope.

\section{Western blot analysis}

HPLSCs were lysed in cell lysis buffer containing $1 \times$ Protease/Phosphatase Inhibitor Cocktail. Protein concentrations were measured with the Pierce BCA Protein Assay Kit. Equal amounts $(15 \mu \mathrm{g})$ of protein along with a protein marker (Precision Plus Protein ${ }^{\mathrm{TM}}$ Western $\mathrm{C}^{\mathrm{TM}}$ Standards) were separated on Mini-PROTEAN ${ }^{\circledR} T G X^{\mathrm{TM}}$ Precast Gels for $30 \mathrm{~min}$ at $200 \mathrm{~V}$. The Trans-Blot ${ }^{\circledR}$ Turbo Transfer System (Bio-Rad Laboratories) was used to transfer the separated proteins to a polyvinylidene fluoride membrane. Western blots were processed using the iBind $^{\mathrm{TM}}$ Western System (Life Technologies, Carlsbad, CA, USA) with primary Abs and horseradish peroxidase-conjugated secondary Abs. $\beta$-actin was used as a loading control. An enhanced chemiluminescence system (SignalFire Plus ECL Reagent) was used to develop the protein bands. The protein levels were quantified by densitometry using the ImageQuant ${ }^{\mathrm{TM}}$ LAS 4000 biomolecular imager (GE Healthcare, Uppsala, Sweden). Band densities were presented as fold-increases of the expression levels of primary Abs (normalized to $\beta$-actin), and compared with the results of control. Quantification results were shown below the corresponding blots. The densitometry data were from a representative of three independent experiments.

\section{Immunocytochemical assay}

After washing with phosphate-buffered saline (PBS), the HPLSCs were fixed with $4 \%$ paraformaldehyde for $10 \mathrm{~min}$ at room temperature (RT), permeabilized with $0.5 \%$ Triton-X in PBS for $10 \mathrm{~min}$, then blocked in $10 \%$ normal goat serum at RT for $10 \mathrm{~min}$ and incubated at $4^{\circ} \mathrm{C}$ with primary Abs (dilution, 1:100). After three washes with PBS for 5 min each, the cells were incubated with the combination of secondary Abs with Alexa Flour 488 or 568 (1:200) and Hoechst $33342(5 \mu \mathrm{g} / \mathrm{ml})$, as the nuclear staining (blue), at RT for $45 \mathrm{~min}$. The stained cells were mounted with ProLong Gold Antifade Mountant and viewed under a light microscope (Keyence Corporation of America, Elmwood Park, NJ, USA).

\section{Results}

\section{Osteogenic differentiation is induced in HPLSCs cultured with OIM}

First, we examined the protein levels of the osteogenesis markers, Runx2 and osterix, and the staining intensity of ALP activity to determine whether OIM can accelerate osteogenic differentiation of HPLSCs. Western blot analysis showed that the expression levels of both Runx2 and osterix were upregulated in HPLSCs cultured in OIM, as compared to without OIM (control) (Fig. 1A). Immunocytochemical analysis showed that Runx2 expression was faintly observed in the cytoplasm of HPLSCs cultured with control medium, whereas HPLSCs with OIM showed intranuclear expression of Runx2 (Fig. 1B). Osterix was also observed in the intranuclear portion of HPLSCs cultured with OIM, even though HPLSCs cultured without OIM showed weakly and diffusely displayed cytoplasmic expression of osterix (Fig. 1B). The intensity of ALP staining was significantly enhanced in HPLSCs cultured in OIM, as compared with control OIM (Fig. 1C). These data demonstrate 
A

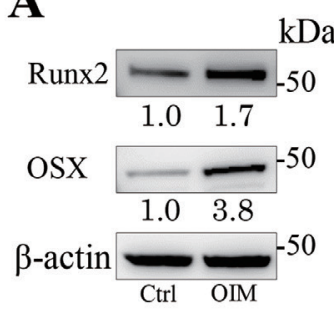

C

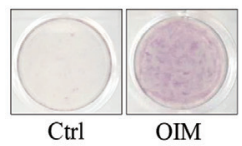

B

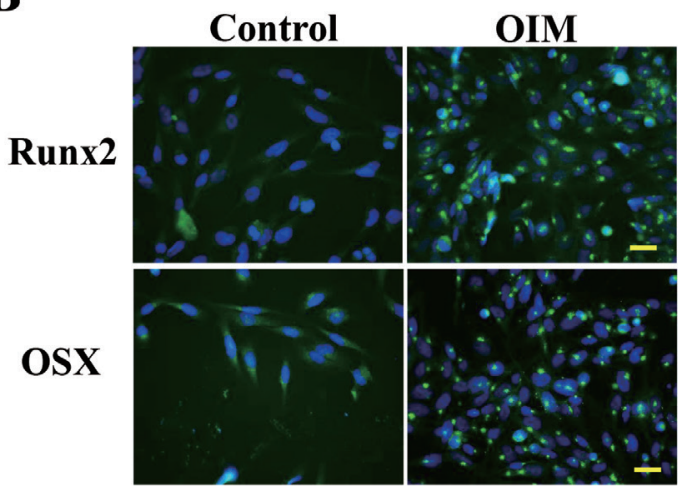

Figure 1. OIM induces osteogenic differentiation of HPLSCs. A-C: HPLSCs were cultured in OIM or control medium for 7 days. A: Western blot analysis of Runx 2 and osterix (OSX) expression in HPLSCs cultured with or without OIM for 7 days. B: Immunocytochemical evaluation of Runx2 and osterix (OSX) expression (green) in HPLSCs cultured with or without OIM. Scale bars, $50 \mu \mathrm{m}$. C: Staining for ALP activity in HPLSCs treated with or without OIM on day 7.

$\mathbf{A}$

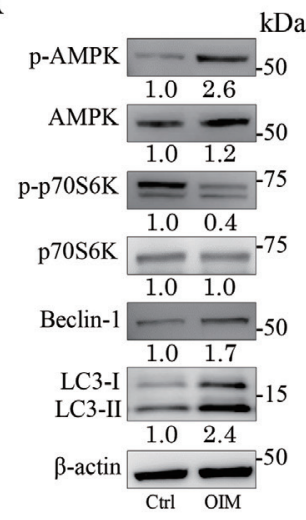

B

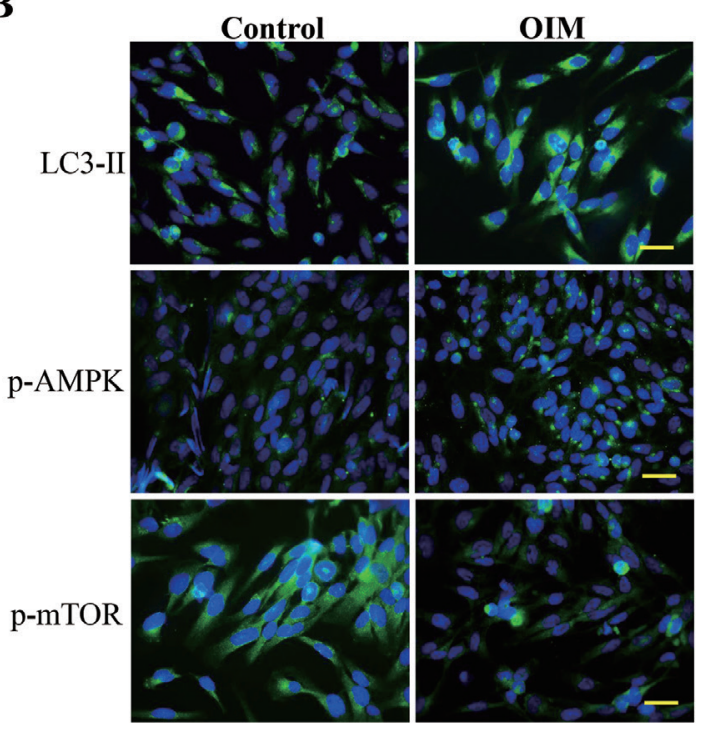

Figure 2. Autophagy induction in HPLSCs cultured with OIM. A and B: HPLSCs were cultured in OIM or control medium for 7 days. A: Expression of the autophagy markers LC3-II and Beclin-1, and the autophagy regulators AMPK and p70S6K in HPLSCs cultured with or without OIM for 7 days, as determined by western blot analysis. B: Immunocytochemical expression (green) of LC3-II, p-AMPK, and p-mTOR in HPLSCs cultured with or without OIM for 7 days. Scale bars, $50 \mu \mathrm{m}$.

that OIM can induce the osteogenic differentiation of HPLSCs.

\section{Autophagy is accelerated in HPLSCs cultured with OIM}

To determine whether OIM can induce autophagy of HPLSCs, we examined the expression levels of autophagy-related factors in cells cultured with or without OIM. The conversion of LC3-I to autophagosome-associated LC3-II was enhanced in HPLSCs cultured with OIM, as determined by western blot analysis (Fig. 2A). By immunocytochemical approaches, many LC3-II-positive autophagosomes were observed in HPLSCs cultured with OIM, as compared to without OIM (Fig. 2B). Conversion changes in LC3-II were correlated with increased expression of the autophagy marker Beclin-1 in HPLSCs cultured with OIM (Fig. 2A). Furthermore, we examined changes in the expression levels of autophagy regulators. The results of western blot analysis showed that the expression of phosphorylated AMPK was increased in HPLSCs cultured with OIM (Fig. 2A), suggesting that the activation of AMPK is related to the induction of autophagy. Immunocytochemical analysis showed that HPLSCs cultured with OIM showed perinuclear expression of phosphorylated AMPK (Fig. 2B). Together, these results indicate that OIM stimulation induced the activation of AMPK in HPLSCs. We used anti-p70S6K and -phosphorylated p70S6K Abs in western blot analysis to evaluate the activation of mTOR. Phosphorylated p70S6K expression was decreased in HPLSCs cultured with OIM, as compared with those cells without OIM (Fig. 2A). Similar to the results of western blotting, the reults of immunocytochemical analysis showed that the expression of phophorylated mTOR was reduced in HPLSCs cultured with OIM, even though remarkable expression of phosphorylated mTOR was observed in the cytoplasm of HPLSCs cultured without OIM (Fig. 2B), indicating the inverse activation of mTOR in HPLSCs cultured with OIM. These findings demonstrate that OIM induced autophagy of HPLSCs 


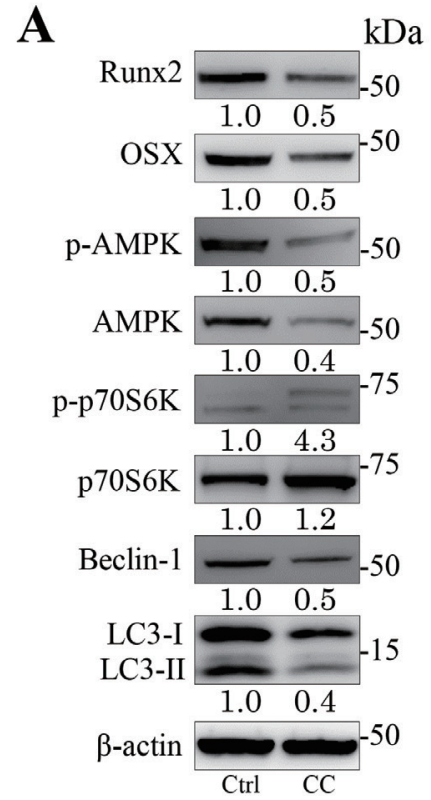

B

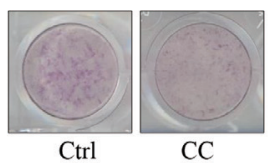

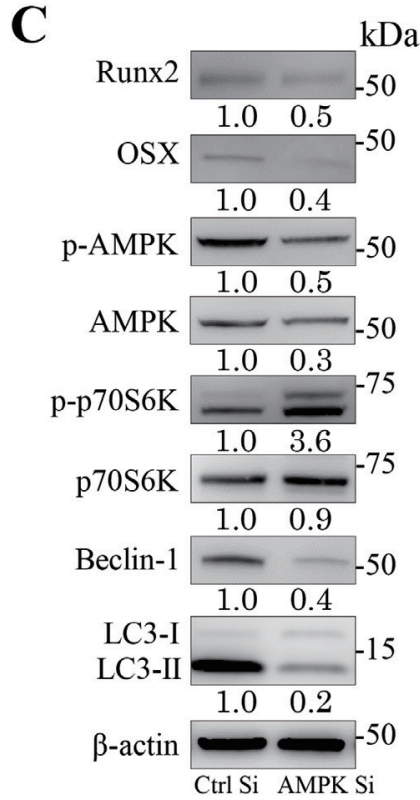

D

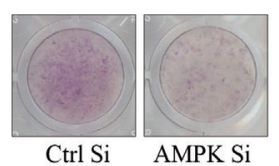

Figure 3. AMPK-related pathways regulate osteogenic differentiation of HPLSCs. A and B: HPLSCs were cultured in OIM with or without the AMPK inhibitor CC $(1 \mu \mathrm{M})$ for 7 days. A: Representative western blots using Abs against AMPK, phosphorylated AMPK (p-AMPK), p70S6K, phosphorylated p70S6K (p-p70S6K), Runx2, osterix (OSX), Beclin-1, and LC3-II. B: Representative images of ALP staining (red) in HPLSCs with or without CC. C and D: HPLSCs were transfected with negative control siRNA (Ctrl Si) or AMPKa siRNA (AMPK Si; $10 \mu \mathrm{M}$ ) for $48 \mathrm{~h}$ before being cultured in OIM for 7 days. C: Western blot analysis of AMPK, phosphorylated AMPK (p-AMPK), p70S6K, phosphorylated p70S6K (p-p70S6K), Runx2, osterix (OSX), Beclin-1, and LC3-II. D: Representative images of ALP staining (red) in HPLSCs transfected with negative control siRNA (Ctrl Si) or AMPK $\alpha$ siRNA (AMPK Si).

through the upregulation of AMPK and downregulation of mTOR.

\section{Attenuation of AMPK activity decreased osteogenic differentiation and autophagy of HPLSCS}

We investigated the role of AMPK in the enhancement of osteogenic differentiation and autophagy of HPLSCs using pharmacological inhibition and genetic knockdown of AMPK. The western blot results showed that the expression levels of both AMPK and phosphorylated AMPK were downregulated in HPLSCs treated with CC (Fig. 3A), indicating that $\mathrm{CC}$ works as AMPK inhibitor. Pharmacological inhibition of AMPK by CC had upregulated expression of phosphorylated p70S6K and decreased expression of LC3-II and Beclin-1, indicating activation of mTOR and reduced autophagic activity (Fig. 3A). CC treatment led to attenuation of osteogenic differentiation of HPLSCs, as demonstrated by the decreased expression of the osteogenic markers Runx2 and osterix, and reduced the intensity of ALP activity (Fig. 3A and B). The efficiency of AMPK $\alpha$ siRNA silencing, but not the control siRNA, was confirmed by decreased expression of both AMPK and phosphorylated AMPK in differentiating HPLSCs at 7 days (Fig. 3C). HPLSCs transfected with AMPK $\alpha$ siRNA showed upregulation of phosphorylated p70S6K, indicating that the inhibition of mTOR activity was relieved by the inactivation of AMPK (Fig. 3C). Decreased expression of the autophagy markers osterix and LC3-II was observed in HPLSCs transfected with AMPK $\alpha$ siRNA (Fig. 3C). Transfection with AMPK $\alpha$ siRNA also suppressed osteogenic differentiation of HPLSCs, as evidenced by downregulated expression of Runx 2 and osterix, and decreased intensity of ALP staining, as compared with that of the cells transfected with the negative control siRNA (Fig. 3C and D). Accordingly, pharmacological inhibition and genetic knockdown of AMPK blocked increased expression of the osteogenic and autophagy markers in HPLSCs, suggesting that AMPK activation may regulate both osteogenic differentiation and acceleration of autophagy of differentiating HPLSCs.

\section{mTOR negatively regulates osteogenic differentiation and autophagy of HPLSCS}

The combination of the mTOR inhibitor rapamycin and its siRNA was used to identify relationships to osteogenic differentiation and autophagy of HPLSCs. The effect of rapamycin pretreatment and mTOR siRNA, but not the control siRNA, was confirmed by suppressed expression of phosphorylated p70S6K in differentiating HPLSCs (Fig. 4A and C). Pharmacological and genetic inhibition of mTOR increased the expression of LC3-II (Fig. 4A and C), indicating that blockade of mTOR leads an acceleration of autophagy activation in differentiating HPLSCs. mTOR inhibition also had upregulated expression of Runx2 and osterix, and increased the intensity of ALP staining in HPLSCs (Fig. 4A-D), indicating enhancement of osteogenic differentiation of HPLSCs by blocking of mTOR activity. These findings suggest that mTOR inhibition may contribute to osteogenic differentiation by inducing autophagy. 

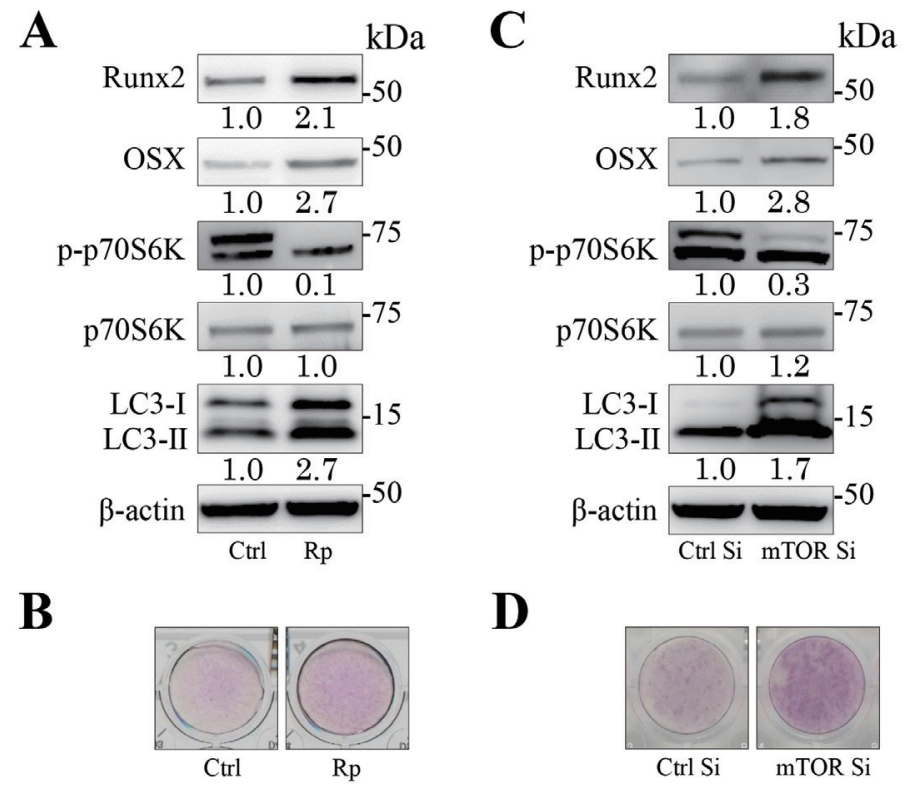

Figure 4. Negative regulation of osteogenic differentiation and autophagy of HPLSCs by mTOR. A and B: After pretreatment with or without rapamycin (Rp; $200 \mathrm{nM}$ ), an inhibitor of mTOR, for $4 \mathrm{~h}$, HPLSCs were cultured with OIM for 7 days. A: Representative western blots using Abs against p70S6K, phosphorylated p70S6K (p-p70S6K), LC3-II, Runx2, and osterix (OSX). B: Representative images of ALP staining (red) in HPLSCs pretreated with or without Rp. C and D: HPLSCs were transfected with negative control siRNA (Ctrl Si) or mTOR siRNA (mTOR Si; $10 \mu \mathrm{M}$ ) for $48 \mathrm{~h}$ before being cultured in OIM for 7 days. C: Western blot analysis of p70S6K, phosphorylated p70S6K (p-p70S6K), LC3-II, Runx2, and osterix (OSX). D: Representative images of ALP staining (red) in HPLSCs transfected with negative control siRNA (Ctrl Si) or mTOR siRNA (mTOR Si).

A

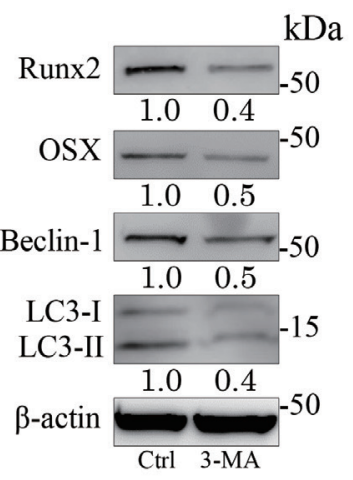

B

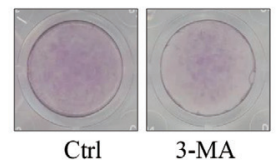

C

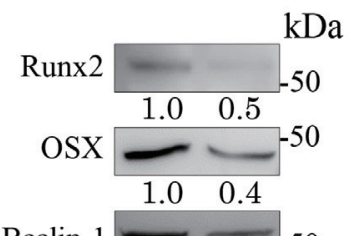

Beclin-1 -50

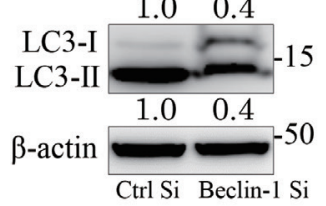

D

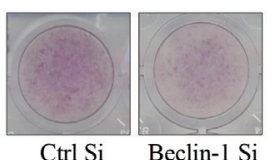

Figure 5. Osteogenesis differentiation of HPLSCs was attenuated after deletion of Beclin-1 by 3-MA and siRNA in HPLSCs. A and B: After pretreatment with or without 3-MA $(5 \mathrm{mM})$ for $4 \mathrm{~h}$, HPLSCs were cultured with OIM for 7 days. A: Representative western blots using Abs against Beclin-1, LC3-II, Runx2, and osterix (OSX). B: Representative images of ALP staining (red) in HPLSCs pretreated with or without 3-MA. C and D: HPLSCs were transfected with negative control siRNA (Ctrl Si) or Beclin-1 siRNA (Beclin-1 Si; $10 \mu \mathrm{M}$ ) for $48 \mathrm{~h}$ before being cultured in OIM for 7 days. C: Western blot analysis of Beclin-1, LC3-II, Runx2, and osterix (OSX). D: Representative images of ALP staining (red) in HPLSCs transfected with negative control siRNA (Ctrl Si) or Beclin-1 siRNA (Beclin-1 Si).

\section{Early stages of autophagy are related to osteogenic differentiation of HPLSCS}

The autophagy process progresses through the early and late stages of osteogenic differentiation of HPLSCs. Therefore, we examined whether the early stages of autophagy participate in osteogenic differentiation of HPLSCs using 3-MA pretreatment, autophagy inhibitors at the early stages, and Beclin-1 siRNA, but not the control siRNA. The influ- ence of these inhibitiors was confirmed by decreased expression of Beclin-1 and LC3-II in HPLSCs (Fig. 5A and C), indicating the suppression of autophagic activity. Inhibiting the early stages of autophagy had reduced expression of the osteogenic markers Runx 2 and osterix, and decreased the intensity of ALP activity in HPLSCs (Fig. 5A-D). These findings suggest that autophagy-related osteogenic differentiation of HPLSCs may be regulated by the early stages of autophagy correspond- 
A

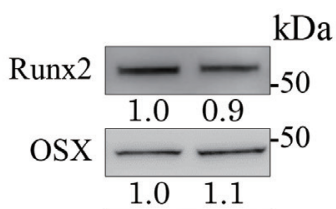

LC3-I

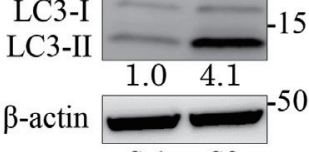

Ctrl CQ

B

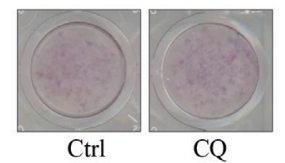

C

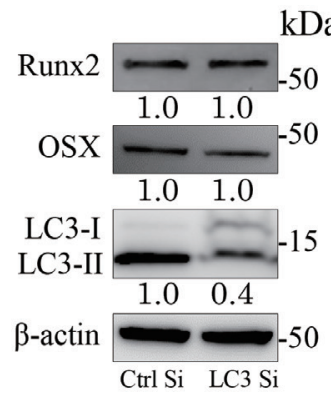

D

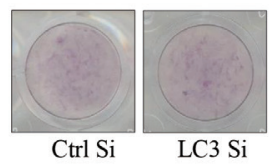

Figure 6. Induction of osteogenic differentiation of HPLSCs was maintained after deletion of LC3-II by CQ and siRNA. A and B: After pretreatment with or without CQ $(10 \mu \mathrm{M})$ for $4 \mathrm{~h}$, HPLSCs were cultured with OIM for 7 days. A: Representative western blots using Abs against LC3II, Runx2, and osterix (OSX). B: Representative images of ALP staining (red) in HPLSCs pretreated with or without CQ. (C and D) HPLSCs were transfected with negative control siRNA (Ctrl Si) or LC3 siRNA ( $\mathrm{LC} 3 \mathrm{Si} ; 10 \mu \mathrm{M})$ for $48 \mathrm{~h}$ before being cultured in OIM for 7 days. C: Western blot analysis of LC3-II, Runx2, and osterix (OSX). D: Representative images of ALP staining (red) in HPLSCs transfected with negative control siRNA (Ctrl Si) or LC3 siRNA (LC3 Si).

ing to Beclin-1 activity.

\section{Inhibition of the late stage of autophagy produced no changes in oste- ogenic differentiation of HPLSCS}

Next, we examined the effect of blockade of the late stage of autophagy on osteogenic differentiation of HPLSCs by the combination of pharmacological inhibition and genetic knockdown of LC3-II. CQ is an inhibitor of the late stages of autophagy and acts to inhibit the fusion of formed autophagosomes and lysosomes. The effects of CQ pretreatment and LC3 siRNA, but not the control siRNA, were verified by decreased expression of LC3-II in HPLSCs (Fig. 6A and C). The late autophagy-inhibited HPLSCs showed no changes in the expression levels of the osteogenic markers Runx 2 and osterix, and the intensity of ALP staining, as compared with control cells (Fig. 6A-D). These findings suggest that, unlike the early stages, the late stages of autophagy may not regulate variation in osteogenic differentiation of HPLSCs.

\section{Discussion}

The results of the present demonstrate a role of autophagy stages in the osteogenic differentiation of HPLSCs. The process of autophagy is generally divided into two parts, the early and late stages. Our results highlight three main points. First, the acceleration of osteogenic differentiation of HPLSCs was regulated by the early stages of autophagy. Second, negative regulation of mTOR contributed to autophagy-induced osteogenic differentiation. Third, activation of AMPK mediated early autophagy-accelerated osteogenic differentiation through the downregulation of mTOR and upregulation of Beclin-1.

The most striking finding is that inhibition of the PI3KcIII/Beclin-1 complex led to decreased enhancement of osteogenic differentiation of HPLSCs. The PI3KcIII/Beclin-1 complex regulates the initiation and nucleation phases of the autophagy process, which are essential for phagophore formation $^{8}$. 3-MA inhibits the early stages of autophagy by blocking autophagosome formation via the inhibition of PI3KcIII ${ }^{20)}$. In contrast, HPLSCs pretreated with CQ or transfected with LC3 siRNA showed no changes in the enhancement of osteogenic differentiation. $\mathrm{CQ}$ is one of the most widely employed inhibitors of the last stage of autophagy in in vitro studies ${ }^{21,22)}$. LC3-II is a component of the ubiquitin-like conjugation systems that ensures the lipidation of autophagosome markers for regulation of the maturation phase of the autophagy process ${ }^{9)}$. These findings of autophagy inhibition suggest that the enhancement of osteogenic differentiation of HPLSCs is regulated by the early stages of autophagy, whereas the late stages had no influence. Similarly, a recent study has postulated the crucial role of early autophagy in the induction of osteogenic differentiation of human $\mathrm{MSCs}^{5}$. In contrast, a few studies revealed that the late stages of autophagy may contribute to the maintenance of bone mass based on the findings that pharmacological and genetic inhibition of late autophagy decreased bone loss in animal models ${ }^{23,24)}$.

Inhibition of mTOR activity represents increased enhancement of autophagy-mediated osteogenic differentiation of HPLSCs. In this study, anti-p70S6K and -phosphorylated p70S6K Abs were used to evaluate the activation of mTOR, which leads to the phosphorylation of the downstream protein $\mathrm{p} 70 \mathrm{~S} 6 \mathrm{~K}^{25,26)}$. Although the mTOR/p70S6K signaling pathway is required for osteoblast proliferation and differentiation, the effect of mTOR activity on osteogenic differentiation remains controversial $^{18,19)}$. Suppression of mTOR signaling with rapamycin has been shown to promote osteogenesis in vitro, as measured by an increase in ALP activity, an increase in mineral formation, and the upregulation of genes involved in osteogenesis ${ }^{18)}$. Our results support those of that study. In the present study, mTOR inhibition also led to increased LC3-II expression. mTOR, under diverse positive conditions, such as high energy levels, amino acids, and growth factors, was found to inhibit autophagy ${ }^{17)}$. Therefore, mTOR may negatively regulate the autophagy-mediated osteogenic differentiation of HPLSCs.

The combination of pharmacological inhibition and gene silencing demonstrated that AMPK-dependent inhibition of mTOR is necessary for the enhancement of osteogenic differentiation of HPLSCs and mediated increased activity of autophagy. Our results are consistent with the ability of AMPK to induce autophagy through inactivation of mTOR in various cell types ${ }^{27}$. AMPK inhibition led to decreased expression of both Beclin-1 and LC3-II, suggesting that AMPK directly regulates the autophagy process. Particularly, our data that AMPK inhibition de- 
creased Beclin-1 activity led us to speculate that AMPK directly regulates the early stage of autophagy. This speculation supports recent studies that AMPK regulates autophagy by activation of Beclin- ${ }^{28,29)}$. Together, these findings suggest that AMPK activation enhances the early stages of autophagy-mediated osteogenic differentiation of HPLSCs via dual mechanisms involving not only inactivation of mTOR, but also direct activation of Beclin-1.

There were some possible limitations to this study, especially the lack of direct evidence of why inactivation of mTOR can accelerate autophagy-mediated osteogenic differentiation. Recent studies proposed that AMPK activation activates autophagy via dual mechanisms involving not only inactivation of mTOR, but also direct phosphorylation of $\mathrm{ULK} 1^{27}$. Therefore, further studies are warranted to examine the involvement of ULK1 activity during osteogenic differentiation of HPLSCs.

In conclusion, this study revealed that AMPK-dependent mTOR inhibition- and Beclin-1 activation-mediated early stages of autophagy are required for the enhancement of osteogenic differentiation of HPLSCs, which may be targeted to advance the osteogenic capacity of MSCs and provide unique strategies for bone regeneration and repair.

\section{Acknowledgments}

We would like to thank Enago (Academic Proofreading Service; www.enago.jp) for the English language review. This work was supported by JSPS KAKENHI (grant number: 18K09567 to JO) and the Private University Research Branding Project.

\section{Conflict of Interest}

The authors have no competing interest to declare.

\section{References}

1. Mizushima N, Levine B, Cuervo AM and Klionsky DJ. Autophagy fights disease through cellular self-digestion. Nature 451: 10691075, 2008

2. Cuervo AM, Bergamini E, Brunk UT, Droge W, Ffrench M and Terman A. Autophagy and aging: the importance of maintaining "clean" cells. Autophagy 1: 131-140, 2005

3. Mizushima N and Levine B. Autophagy in mammalian development and differentiation. Nat Cell Biol 12: 823-830, 2010

4. Wan Y, Zhuo N, Li Y, Zhao W and Jiang D. Autophagy promotes osteogenic differentiation of human bone marrow mesenchymal stem cell derived from osteoporotic vertebrae. Biochem Biophys Res Commun 488: 46-52, 2017

5. Pantovic A, Krstic A, Janjetovic K, Kocic J, Harhaji-Trajkovic L, Bugarski D and Trajkovic V. Coordinated time-dependent modulation of AMPK/Akt/mTOR signaling and autophagy controls osteogenic differentiation of human mesenchymal stem cells. Bone 52: 524-531, 2013

6. Mizushima N. Autophagy: process and function. Genes Dev 21: 2861-2873, 2007

7. Funderburk SF, Wang QJ and Yue Z. The Beclin 1-VPS34 complex-at the crossroads of autophagy and beyond. Trends Cell Biol 20: 355-362, 2010

8. McKnight NC and Zhenyu Y. Beclin 1, an essential component and master regulator of PI3K-III in health and disease. Curr Pathobiol Rep 1: 231-238, 2013

9. Kabeya Y, Mizushima N, Ueno T, Yamamoto A, Kirisako T, Noda T, Kominami E, Ohsumi Y and Yoshimori T. LC3, a mammalian homologue of yeast Apg8p, is localized in autophagosome mem- branes after processing. EMBO J 19: 5720-5728, 2000

10. Krampera M, Pizzolo G, Aprili G and Franchini M. Mesenchymal stem cells for bone, cartilage, tendon and skeletal muscle repair. Bone 39: 678-683, 2006

11. Chen Y, Shao JZ, Xiang LX, Dong XJ and Zhang GR. Mesenchymal stem cells: a promising candidate in regenerative medicine. Int J Biochem Cell Biol 40: 815-820, 2008

12. Trubiani O, Di Primio R, Traini T, Pizzicannella J, Scarano A, Piattelli A and Caputi S. Morphological and cytofluorimetric analysis of adult mesenchymal stem cells expanded ex vivo from periodontal ligament. Int J Immunopathol Pharmacol 18: 213-221, 2005

13. Wang L, Shen H, Zheng W, Tang L, Yang Z, Gao Y, Yang Q, Wang C, Duan $Y$ and Jin Y. Characterization of stem cells from alveolar periodontal ligament. Tissue Eng Part A 17: 1015-1026, 2011

14. Fujii S, Maeda H, Wada N, Tomokiyo A, Saito M and Akamine A. Investigating a clonal human periodontal ligament progenitor/stem cell line in vitro and in vivo. J Cell Physiol 215: 743-749, 2008

15. Khoshhal M, Amiri I and Gholami L. Comparison of in vitro properties of periodontal ligament stem cells derived from permanent and deciduous teeth. J Dent Res Dent Clin Dent Prospects 11: 140148, 2017

16. Liu J, Li Q, Liu S, Gao J, Qin W, Song Y and Jin Z. Periodontal ligament stem cells in the periodontitis microenvironment are sensitive to static mechanical strain. Stem Cells Int 2017: 1380851, 2017

17. Alers S, Loffler AS, Wesselborg S and Stork B. Role of AMPKmTOR-Ulk1/2 in the regulation of autophagy: cross talk, shortcuts, and feedbacks. Mol Cell Biol 32: 2-11, 2012

18. Lee KW, Yook JY, Son MY, Kim MJ, Koo DB, Han YM and Cho YS. Rapamycin promotes the osteoblastic differentiation of human embryonic stem cells by blocking the mTOR pathway and stimulating the BMP/Smad pathway. Stem Cells Dev 19: 557-568, 2010

19. Singha UK, Jiang Y, Yu S, Luo M, Lu Y, Zhang J and Xiao G. Rapamycin inhibits osteoblast proliferation and differentiation in MC3T3-E1 cells and primary mouse bone marrow stromal cells. J Cell Biochem 103: 434-446, 2008

20. Wu YT, Tan HL, Shui G, Bauvy C, Huang Q, Wenk MR, Ong CN, Codogno P and Shen HM. Dual role of 3-methyladenine in modulation of autophagy via different temporal patterns of inhibition on class I and III phosphoinositide 3-kinase. Journal Biol Chem 285: 10850-10861, 2010

21. Redmann M, Benavides GA, Berryhill TF, Wani WY, Ouyang X, Johnson MS, Ravi S, Barnes S, Darley-Usmar VM and Zhang J. Inhibition of autophagy with bafilomycin and chloroquine decreases mitochondrial quality and bioenergetic function in primary neurons. Redox Biol 11: 73-81, 2017

22. Mauthe M, Orhon I, Rocchi C, Zhou X, Luhr M, Hijlkema KJ, Coppes RP, Engedal N, Mar M and Reggiori F. Chloroquine inhibits autophagic flux by decreasing autophagosome-lysosome fusion. Autophagy 14: 1435-1455, 2018

23. Dai $\mathrm{Y}$ and $\mathrm{Hu} \mathrm{S}$. Recent insights into the role of autophagy in the pathogenesis of rheumatoid arthritis. Rheumatology (Oxford) 55: 403-410, 2016

24. Lin NY, Chen CW, Kagwiria R, Liang R, Beyer C, Distler A, Luther J, Engelke K, Schett G and Distler JH. Inactivation of autophagy ameliorates glucocorticoid-induced and ovariectomy-induced bone loss. Ann Rheum Dis 75: 1203-1210, 2016

25. Inoki K, Mori H, Wang J, Suzuki T, Hong S, Yoshida S, Blattner SM, Ikenoue T, Hall MN, Kwiatkowski DJ, rastaldi MP, Huber TB, Kretzler M, Holzman LB, Wiggins RC and Guan KL. mTORC1 ac- 
tivation in podocytes is a critical step in the development of diabetic nephropathy in mice. J Clin Invest 121: 2181-2196, 2011

26. Lieberthal $\mathrm{W}$ and Levine JS. The role of the mammalian target of rapamycin (mTOR) in renal disease. J Am Soc Nephrol 20: 24932502, 2009

27. Hardie DG. AMPK and autophagy get connected. EMBO J 30: 634635, 2011

28. Zhang D, Wang W, Sun X, Xu D, Wang C, Zhang Q, Wang H, Luo W,
Chen Y, Chen H and Liu Z. AMPK regulates autophagy by phosphorylating BECN1 at threonine 388. Autophagy 12: 1447-1459, 2016

29. He C, Zhu H, Li H, Zou MH and Xie Z. Dissociation of Bcl-2-Beclin1 complex by activated AMPK enhances cardiac autophagy and protects against cardiomyocyte apoptosis in diabetes. Diabetes 62 : 1270-1281, 2013 